https://doi.org/10.5719/aub-g/69.1/7

\title{
MODÉLISATION PRÉDICTIVE DE L'ÉTALEMENT URBAIN DES GRANDES MÉTROPOLES DU BASSIN MÉDITERRANÉEN SUR L'ESPACE AGRICOLE : APPLICATION AU SECTEUR ORIENTAL DE L'AGGLOMÉRATION ALGÉROISE (ALGÉRIE)
}

\author{
MOHAMED ATIK ${ }^{1}$, ABDELKADER ABDELLAOUI ${ }^{2}$, \\ RACHID NEDJAI ${ }^{3}$, BELKACEM BELAL $^{4}$
}

\begin{abstract}
Résumé
Plusieurs régions algériennes connaissent depuis quelques années des transformations spectaculaires et de façon très rapide, d'un paysage rural à une urbanisation massive avec d'un mode de vie moderne en remplacement du mode de vie traditionnel et campagnard. Ce phénomène est encore plus ressenti au niveau des périphéries des grands centres urbains. La ville d'Alger en est une illustration frappante ; elle a connu en effet une croissance urbaine sans précédent ces quarante dernières années (1972-2012).

Depuis la fin des années 1980, cette croissance urbaine prend la forme d'un mouvement de périurbanisation, qui s'est traduit par une consommation excessive des terres à hautes potentialités agricoles en dépit d'un dispositif législatif mis en place après l'indépendance pour préserver le patrimoine agricole.

L'objectif de cette étude est d'analyser les changements d'occupation du sol à partir d'imagerie satellitale et de données terrain. La modélisation de l'évolution de l'occupation à l'aide du modèle LCM (Land Change Modeler) et par l'application des méthodes MLP et RegLog confirme la poursuite de la substitution des terres agricoles essentiellement par du bâti jusqu'en 2020, suite à quoi un ralentissement s'amorce pour se stabiliser autour de 2030 en raison du déficit du foncier qui arrive à manquer.

Mots clés: Croissance urbaine, périurbanisation, foncier agricole, images satellitaires, carroyage, classification supervisée, modèle LCM, occupation du sol.

1 ESEAD -FLASH Université Ibn Zohr Agadir - Associer CEMOTEV, Université de Versailles Saint-Quentin-en-Yvelines, mohamed.atik@orange.fr

2 AVCN Paris, abdellaoui.geo@gmail.com

3 CEDETE - Université d'Orléans.

4 ENS Bouzaréah Alger.
\end{abstract}




\section{Introduction}

Plusieurs régions algériennes connaissent depuis quelques années des transformations spectaculaires passant, de façon très rapide, d'un paysage rural à une urbanisation massive avec un mode de vie moderne en remplacement du mode de vie traditionnel et campagnard. Le développement de la civilisation urbaine est aujourd'hui une donnée incontournable de cette situation nouvelle où la ville et l'urbanité s'emparent du territoire et le transforment tandis que les rapports sociaux se complexifient en même temps que se transforme la société. La problématique de l'usage du sol doit désormais être placée au cœur du débat sur l'aménagement du territoire pour au moins trois raisons qui sont i) la forte croissance démographique, ii) l'amenuisement considérable des surfaces agricoles et iii) l'apparition continue de grands complexes urbains ne répondant ni aux normes de développement durable ni aux attentes des citoyens dont l'habitude d'habiter a complètement changé au cours des quinze dernières années.

La ville d'Alger, capitale et grande métropole, a connu une croissance urbaine sans précédent ces quarante dernières années (1972-2017). La maîtrise de cette extension devrait constituer un enjeu majeur. Le tableau (1) nous montre à titre indicatif l'évolution démographique de l'Algérie entre 1966 et 2018, avec une projection pour 2040 ainsi que celle de la wilaya d'Alger selon les quatre derniers recensements (entre 1977 et 2008). Nous noterons que la population a presque doublé entre 1977 et 2008 (rapport égal 1. 88).

\begin{tabular}{|c|c|}
\hline année & population en milliers \\
\hline 1966 & 12096 \\
\hline 1970 & 13309 \\
\hline 1975 & 15768 \\
\hline 1980 & 18666 \\
\hline 1985 & 21863 \\
\hline 1990 & 25022 \\
\hline 1995 & 28060 \\
\hline 2000 & 30879 \\
\hline
\end{tabular}


MODÉLISATION PRÉDICTIVE DE L'ÉTALEMENT URBAIN DES GRANDES MÉTROPOLES DU BASSIN MÉDITERRANÉEN SUR L’ESPACE AGRICOLE :...

\begin{tabular}{|c|c|}
\hline 2005 & 32906 \\
\hline 2010 & 35978 \\
\hline 2018 & 42578 \\
\hline 2040 & 57625 \\
\hline & \\
\hline \multicolumn{2}{|c|}{ wilaya d'Alger } \\
\hline 1977 & 1588 \\
\hline 1987 & 1690 \\
\hline 1998 & 2562 \\
\hline 2008 & 2988 \\
\hline
\end{tabular}

Sources : données et projection ONS

Pourtant, depuis l'indépendance l'Algérie a déployé de multiples efforts pour la valorisation et la protection $\mathrm{du}$ foncier agricole. L'aménagement du territoire a pris en charge dans ses objectifs la préservation des terres à fort potentiel agricole; de nombreuses lois ont été promulguées, plusieurs plans d'aménagements du territoire ont été mis en œuvre pour la protection des terres agricoles et l'interdiction de toutes atteintes à ce patrimoine vital. Ainsi depuis la promulgation de La Loi $\mathrm{n}^{\circ} 25-90$ portant sur l'orientation foncière les politiques d'aménagement s'orientent désormais vers des objectifs de développement durable, notamment en termes de préservation d'espaces naturels et agricoles. La loi d'orientation agricole 08-16 du 03 août 2008 est venue consolider les principes énoncés par la loi d'orientation foncière pour la préservation et la valorisation du patrimoine foncier. La réalité terrain est pourtant bien différente et les plans d'aménagement urbain et les plans d'occupation du sol sont vite dépassés par des réalisations effectives bien différentes et des réalités sociales en constante mutation et dont il n'est pas toujours tenu compte.

Les mesures, au demeurant louables, se sont avérées, avec le temps, peu efficaces et insuffisantes car déconnectées d'un contexte social difficile à cerner en l'absence d'études sociologiques sérieuses et disponibles. On assiste ainsi depuis plus de trois décennies à une dilapidation effrénée des terres à haute potentialité agricole comme le souligne le Conseil National Economique et Social (CNES) dans son rapport sur le foncier en 2004. Déjà depuis 1997, le CNES avait alerté les pouvoirs publics, dans son rapport sur l'environnement, sur les problèmes de la 
préservation des terres agricoles face aux effets de la croissance rapide de la population et des programmes de développement socio-économiques en citant des chiffres alarmants sur la dégradation du foncier agricole. (CNES, 1997).

L'utilisation du sol et la préservation des ressources naturelles ont fait l'objet de nombreux travaux de recherches et développement notamment durant la seconde moitié du $20^{\text {ième }}$ siècle; les premières études ont été faites aux Etats-Unis dans les années 1930 (Marius T. et al, 2011) puis se sont généralisées par la suite. Dès 1956 quelques applications de cartographie de l'utilisation du sol ont été présentées au 18 congrès international de géographie au Brésil (George Pierre, 1957) ; d'autres travaux sont réalisés en Pologne (Dziewonski M, 1956), en Italie (Gribandi D. et Abani D., 1956) et au Pakistan (Ahmad M.N., 1956).

Au Canada des études pionnières ont été menées durant les années 60 et 70 (Clibbon et Gagnon 1965; Munn et al. 1966 ; Tomlinson 1967 ; Coombs et Thie 1979). En Europe le suivi des changements d'occupation du sol est assuré par le projet Corine Land Cover pour 38 états européens (1985). Cet inventaire, issue de l'interprétation visuelle d'images satellitaires, avec des données complémentaires d'appui, fournit une information géographique de référence sur les changements d'occupation du sol dans les pays européens (European Environment Agency, 2000).

Les images satellitaires multi dates disponibles depuis le lancement du programme ERTS début des années 1970 ont permis de réaliser des analyses spatio-temporelles pour le suivi et la modélisation des changements de l'utilisation du sol (Hung 2000 ; Pagelow 2004); nous pouvons également citer les travaux de Langran 1992, Peuquet 1994, Claramunt et Theriault 1995, 1996, Claramunt et al, 1999, Wachowicz 1999, Dragicevic et Marceau 2000, Hornsby et Yuan 2008. La mise en évidence du construit par images satellitaires a été moins étudiée que le thème végétation par exemple où une multitude d'indices ont été développés (Bannari, 1996) ; nous pouvons citer cependant les travaux de Abdellaoui et al. (1995, 2007 et 2010) sur la proposition d'un indice du bâti et son application.

Pour détecter le changement d'occupation de l'espace sur la période 1987-2005, nous avons dans un premier temps, déterminé pour chaque date l'espace bâti et l'espace agricole par classification 
supervisée. La seconde étape est plus orientée vers la prédiction et par conséquent le devenir de cette partie Est de l'Algérois face à cette situation complexe où les terres agricoles voient de jour en jour leur surface se réduire en particulier sur les trente dernières années. Pour évaluer l'acuité du problème et mettre en exergue l'état futur du secteur d'étude, une approche modélisatrice a été pratiquée par l'utilisation du modèle LCM (Land Change Modeler) et l'application des deux méthodes : MLP et RegLog implémentées dans Idrisi (Eastman, 2006). Deux cartes prédictives respectivement pour 2020 et 2030 ont été élaborées à partir des photos satellites de 2001 et 2005, considérées comme images de références.

\section{Zone d'étude}

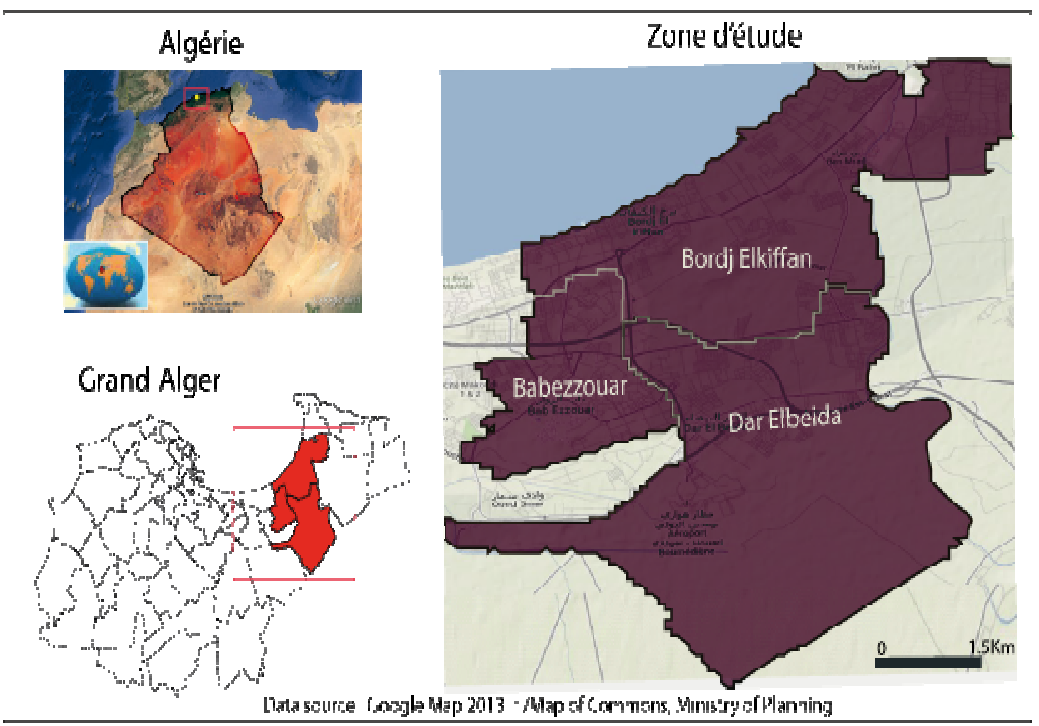

Fig. 1. Carte de la localization de la zone d'etude

Pour cette étude nous avons choisi trois communes dans la périphérie Est d'Alger (Bordj el Kiffan, Bab-Ezzouar et Dar el Beida) sur une superficie de $63 \mathrm{~km}^{2}$; la figure 1 donne la localisation de la zone d'étude. Elle est significative de la croissance urbaine très rapide et 
d'une forte consommation des surfaces agricoles malgré l'existence de règles strictes de l'urbanisme et de la préservation du foncier agricole. Les densités de population sont respectivement pour les trois communes de Bordj el Kiffan, Bab Ezzouar et Dar el Beida de 7002, 11737 et 2404 hab/km2 ; ces valeurs sont extraites du RGPH 2008.

La zone est caractérisée par une pluviométrie relativement abondante (700mm en moyenne par an) mais mal repartie dans le temps et marquée par une opposition entre une saison froide et humide et une saison chaude et sèche (Mutin, 1997). Les températures maximales peuvent atteindre $32^{\circ} \mathrm{C}$ en été et les minimales ne dépassent pas $5^{\circ} \mathrm{C}$. La région est exposée aux vents chauds comme le siroco qui souffle du sud vers le nord à la fin du printemps et pendant l'été ce qui induit une évapotranspiration intense et augmente les besoins en eau des plantes. Les sols qui couvrent la totalité de la zone d'étude ont une grande aptitude culturale; il s'agit de sols profonds riches en matières minérales qui se sont développés sur des alluvions récents du quaternaire et conviennent pour plusieurs cultures. Mais c'est à l'irrigation que cette région doit l'important développement des productions maraîchères et arboricoles.

\section{Méthodes et outils}

\section{Les données}

Pour le présent travail, nous avons utilisé : trois images satellitales Landsat (21 juin 1984, 16 avril 2001 et 21 juin 2005), deux photos aériennes respectivement de 1992 et 1998 à l'échelle de 1/40000ième, quatre cartes topographiques au 1/25000ième (Alger Est (13-4-31), Alger Ouest (14-4-31), Rouiba Est (31-4-31) et Rouïba Ouest (32-431)), la carte des limites administratives sous MapInfo, les données de recensements de la population (RGPH 1987, 1998 et 2008) et les plans d'urbanisme (PDAU d'Alger).

Les traitements des images satellitaires sont réalisés sous IDRISI et ENVI; la base de données spatio-temporelle pour suivre les changements d'occupation du sol dans la zone d'étude est élaborée sous ArcGIS. 


\section{La démarche}

\subsection{Classification supervisée}

\section{i. Prétraitement des images}

Le prétraitement regroupe les processus de ré-échantillonnage, la sélection de la zone d'intérêt, le masquage des zones d'eau. Il est conçu pour améliorer la qualité des images en les rendant aptes à l'interprétation.

Les images utilisées étant de résolutions différentes, nous réalisons une opération de ré-échantillonnage en appliquant la technique de convolution cubique par approximation bicubique de 16 voisins les plus proches du pixel concerné ; ceci permet de modéliser l'image localement par une surface polynomiale (Abdellaoui et Rougab, 1995). Nous obtenons alors des plans spectraux qui vont coïncider parfaitement, au niveau de chaque pixel, avec l'image de référence (images de 2005). La précision de ces transformations varie selon les images; dans notre cas l'opération a produit une erreur moyenne de calibrage (Root Mean Square Error) de 0,30, ce qui est acceptable (Moller-Jensen, 1990). L'extraction de la zone d'étude a été réalisée par un découpage basé sur la technique des coordonnées géographiques.

L'image initiale est peu lisible car peu contrastée ; la plupart des pixels se retrouvent sur une plage réduite de l'échelle de codage $(0 ; 255)$. Un étalement de la dynamique est alors nécessaire pour rendre les différents canaux plus lisibles et donc de reconnaître les principaux thèmes présents sur la scène d'étude. Par la suite, nous avons délimité l'emprise urbaine en transformant nos images de base en images binaires en codant les pixels par des valeurs : 1 pour l'emprise urbaine et 0 pour autre occupation de l'espace. Un seuillage a été réalisé pour isoler l'unité urbaine car il est très utile d'exclure la végétation et l'eau (mer et rivière) des zones urbaines afin de réduire le temps de calcul et d'éviter toute confusion entre la végétation, le sol nu et le bâti.

Nous avons également effectué une étude diachronique pour mettre en évidence les changements d'occupation du sol puis un filtrage par filtre alterné séquentiel pour améliorer l'image.

La méthode de classification non dirigée du maximum de vraisemblance requiert des prétraitements apportés aux différentes images 
pour les rendre le plus comparable possible ; des sites d'entraînement et de validation de qualité doivent être également disponibles pour assurer l'exactitude des classifications obtenues (Jensen, 1996).

\section{ii. La classification supervisée des zones urbaines}

Cette méthode suppose la définition d'aires d'apprentissage pour chacune des classes de référence (bâti, végétation, sol nu) avec une distribution gaussienne (Jensen, 1996). Pour respecter la distribution normale, nous avons employé, dans la sélection des aires d'apprentissage, les groupes obtenus dans la classification non supervisée avec l'algorithme ISOCLUST. Ainsi nous garantissons la sélection d'aires homogènes. Le principe de la classification Bayésienne étant de segmenter une image par le calcul de probabilités d'appartenance, pour chaque pixel de l'image, à l'une des trois classes définies par des zones d'apprentissages (bâti, végétation, sol nu) (Robin, 2002). Dans de nombreux travaux de classification, la bande 7 (moyen infrarouge) est utilisée conjointement aux autres bandes $(1,2,3,4,5,7)$ (JENSEN, 1996).

L'hétérogénéité des objets urbains et du sol nu (routes, carrières, roches...) rend difficile la constitution de classes thématiques représentatives. L'analyse visuelle des classifications relatives aux différentes dates a détecté certaines indications du changement du bâti au non bâti qui ne correspondaient pas à la réalité du terrain. De même, le résultat du traitement a permis d'isoler l'emprise urbaine pour chaque date.

Pour une étude diachronique, nous avons superposé et combiné les images de 1987 et 2001 puis 2001 et 2005 et enfin 1987 et 2005 ; dans ces combinaisons, l'interprétation est beaucoup plus simple. Le résultat final est conforme à la réalité observée sur le terrain et aux références de bases (cartes topographiques, photographies aériennes...). Les couleurs bleue, rouge et vert symbolisent l'évolution à partir des trois dates : 1987, 2001 et 2005. Après insertion manuelle des réseaux viaires, les résultats se sont révélés assez satisfaisants et correspondent aux données du terrain. 


\subsection{Modélisation prédictive de l'occupation des sols}

La prédiction de l'occupation des sols peut être approchée par l'une des techniques suivantes : modèles mathématiques, statistiques d'évolution (systèmes multi-agents), cellulaires (automates cellulaires) (Hubert-Moy et al., 2006) et les modèles basés sur une approche dite "approximative" (Okwuashi et al, 2012; He et al., 2006; Huang et al., 2008 ; Hepinstall et al., 2008; Hubert-Moy et al., 2006). Un large panel de ces méthodes ont été implémentés pour aboutir soit à des applications autonomes soit intégrés dans des applications SIG à l'image LCM (Land Change Modeler) dans Idrisi ou ArcGis, chaîne de Markov, CA-Markov et GEOMOD. Ces méthodes sont par conséquent considérées comme performantes pour la prédiction de l'évolution de l'occupation des sols en raison de leur couplage aux SIG et l'utilisation de leurs potentiels de traitement, de simulation écologique (Eastman, 2006; Pontius et Chan, 2006; Tewolde et Cabral, 2011). La cartographie prédictive de l'occupation des sols de l'est algérois a été réalisée à l'aide du module LCM (Land Change Modeler) intégrée dans le logiciel Idrisi de la version Taiga (Eastman, 2006). Une liste de variables dites explicatives a été établie pour servir de données d'entrée au module LCM et le calcul de la transition d'une catégorie de l'occupation vers d'autres sous forme d'un traitement séquentiel donnant lieu au final à des représentations à la fois graphiques et cartographiques. Ces transitions permettent à la fois d'évaluer la validité du modèle soit en se basant sur un tableau croisé (méthode dite visuelle) soit par une approche statistique en se basant sur des coefficients tel que ROC, Kappa. Pour cela, les missions satellitales précédemment utilisées servent à la fois à l'élaboration de cartes d'occupation des sols et comme cartes de référence sur lesquelles repose toute la phase de validation des cartes prédictives issues de l'application du modèle LCM. Nous avons rajouté au lot de photographies précédentes la mission de 2013 pour consolider la vérification et donc la validité du modèle. Pour cela, les images de 2005 et 2013 serviront à la génération de matrice de confusion et donc à la comparaison des cartes issues du modèle aux cartes issues du traitement des photos satellites. 


\section{Résultats et discussion}

\section{III.1. Les résultats de classification}

Les résultats de classification automatique nous donnent un aperçu des changements d'occupation du sol dans la zone d'étude. L'extension du bâti au détriment des sols agricoles arables est préoccupante durant la période 1987-2005 ; les surfaces bâties passent de 2009 ha en 1987 à 3181 ha en 2005 soit une augmentation de 1172 ha au dépend de la surface agricole en 18 ans, soit une disparition de plus de 65 ha/an de surface agricole en moyenne sur cette période. Ce résultat est illustré par la figure 2.

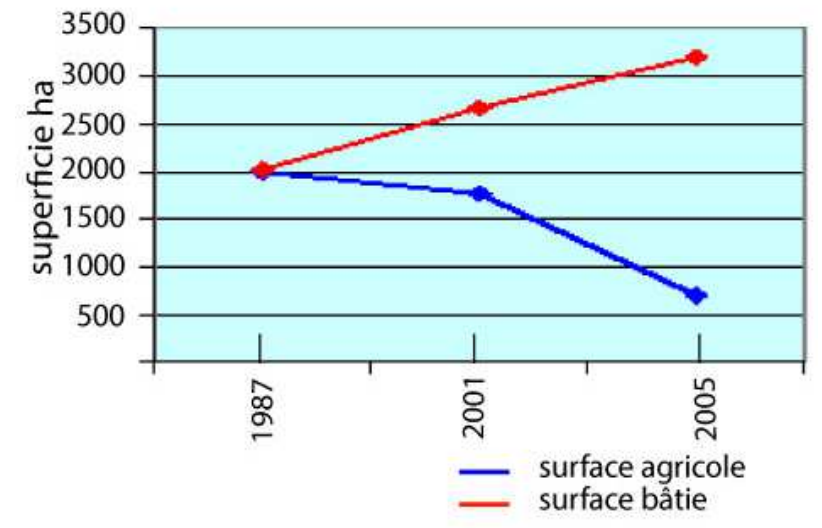

Fig. 2. Occupation du sol entre 1987 et 2005

Nous avons constaté par ailleurs que les espaces urbanisés sont situés sur d'anciennes jachères (des terres qui ne sont pas cultivées de façon permanente).

La figure 3 montre bien la croissance urbaine au détriment des terres agricoles dans la zone d'étude. 


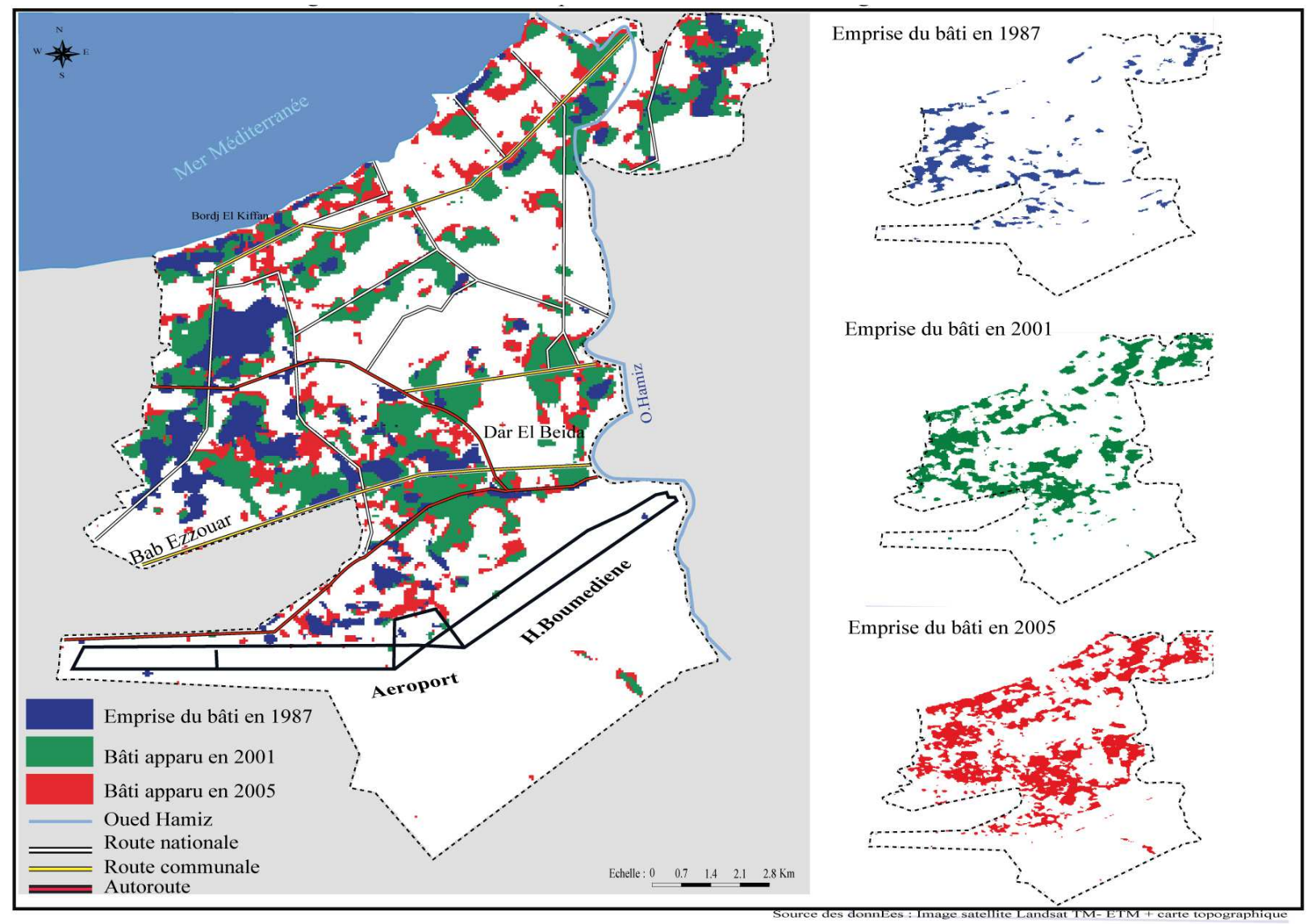

Fig. 3. Chronological evolution of the urban area in Algiers between 1987 and 2005 
Les surfaces urbanisées entre 1987 et 2005 représentent plus de $18 \%$ de la surface totale de la zone et environ (48\%) de la surface agricole selon la définition de la direction des services agricoles d'Alger (tableau 2)

Tableau 2

Extension du bâti de 1987 à 2005

\begin{tabular}{|c|c|c|}
\hline \multicolumn{3}{|c|}{ Pertes des surfaces agricoles par commune } \\
\hline Commune & Perte en sol agricole & \% superficie commune \\
\hline Bordj el Kifan & 639,67 & 54 \\
\hline Dar el Beida & 443 & 37 \\
\hline Bab Ezzouar & 200 & 15,44 \\
\hline
\end{tabular}

\begin{tabular}{|c|c|}
\hline \multicolumn{2}{|c|}{ Récapitulatif sur les trois communes } \\
\hline Surface totale (ha) & 6322 \\
\hline Surface agricole 1988 (ha) & 2459,98 \\
\hline S/ urbanisées 1987-2001 (ha) & 655 \\
\hline S/urbanisées 2001-2005 (ha) & 517 \\
\hline S totale urbanisée 1987-2005 & 1172 \\
\hline \% surface totale & 18,54 \\
\hline \% surface agricole & 47,64 \\
\hline
\end{tabular}

On note sur ce tableau l'accroissement de la consommation des terres agricoles dans les trois communes étudiées pendant la période (1987-2005). L'étalement urbain à empiété sur 1172 ha de terre cultivables en l'espace de 18 ans soit 71 ha par an; on constate ainsi une perte de terres agricoles de plus de $50 \%$ ( $52 \%$ selon nos calculs).

La commune de Bordj El Kiffan, la plus marquée par les constructions illicites, a perdu 639.67 ha soit $54 \%$ de sa superficie agricole et presque la moitié de la surface urbanisée dans la zone d'étude. La plupart de ses déperditions ont eu lieu entre 1990 et 1995, période de l'instabilité sécuritaire en Algérie selon les déclarations des spécialistes du domaine agricole de la commune.

La commune de Dar El Beida a enregistré une diminution de sa superficie agricole de 443 ha soit $37 \%$ de son potentiel agricole et ce en 
dépit des zones protégées aux alentours de l'aéroport d'Alger. La consommation d'espace agricole est de 200 ha à Bab Ezzouar ; comparée à celle des autres communes elle ne représente que $15.44 \%$ de la superficie totale urbanisée dans la zone d'étude. Mais en réalité cette commune a perdu tout son foncier agricole au profit de la réalisation des ZUHN pour satisfaire les besoins croissants de la population algéroise en matière de logement.

Les principaux facteurs de la consommation excessive de l'espace agricole

Diverses raisons expliquent la consommation excessive du foncier agricole dans la zone d'étude :

\section{La période de $1980-1990$}

C'est une dernière période de l'économie dirigée caractérisé par :

A. des flux migratoires intenses de la campagne vers les pôles industriels implantés à la périphérie est d'Alger dans les zones (RouibaReghaia et Harrach - Oued Smar et Sidi Moussa) ; ces flux ont engendré une croissance démographique rapide suivie du développement du bâti destiné à l'habitat individuel. La population de la zone d'étude a ainsi été multipliée par 3.5 en l'espace de 20 ans, passant de 68100 en 1977 à 240600 habitants en 1998 avec un taux d'accroissement très élevé (4 à 6\% en moyenne par an) par rapport au taux d'accroissement national qui n'a pas dépassé $1.6 \%$ en 2008 .

B. de nombreux programmes de logement. Plusieurs programmes d'habitats collectifs ont été réalisés dans les communes de Bab Ezzouar, Dar El Beida et Bordj el Kiffan, parmi lesquels nous pouvons citer :

- La procédure des ZUHN (Zones d'Habitat Urbain Nouvelles) mise en œuvre en 1975.

La totalité de la surface agricole de Bab Ezzouar a été sacrifiée au profit de ce programme pour la réalisation de 10000 logements sur une superficie de 341.45 ha de terre arable.

- Le programme de lotissements individuels lancé les années 1970, mal contrôlé et pas suffisamment cadré, a été plus consommateur d'espace vu sa propagation horizontale et son développent anarchique ; plus 77.129 ha sur la commune de Bordj el Kiffan ont été pris aux 
surfaces agricoles (Service d'urbanisme de la commune de Bordj El Kiffan, 1994).

\section{La période $1990-2000$}

C'est une phase particulière de l'histoire de l'Algérie qui se distingue par la détérioration de la situation sécuritaire induisant à la fois i) de nouvelles vagues d'exode rurale vers les zones périphériques des concentrations urbaines et en particulier d'Alger et ii) des difficultés de contrôle et d'application de la réglementation en matière d'urbanisme ouvrant ainsi encore plus la porte à la consommation anarchique du foncier agricole.

\section{La période 2001-2005}

Deux catastrophes naturelles ont touché Alger et sa périphérie Est : l'accident météorologique du 10 novembre 2001 qui a provoqué les glissements de terrain et les écoulements de boue de Bab El Oued et le séisme de Boumerdés du 21 mai 2003. Pour reloger les sinistrés, les autorités avaient installé des chalets en préfabriqué sans étude préalable sur des terres agricoles ; plusieurs hectares de terres arables ont été ainsi artificialisés.

\section{III.2. Cartographie prédictive de l'évolution de l'occupation des sols de la banlieue Est d'Alger}

Les résultats de l'analyse précédente ont été utilisés pour la modélisation de l'évolution de l'occupation des sols et plus particulière la catégorie "bâti » dans la partie Est du département d'Alger. Ce secteur, connu pour son agriculture maraichère subit les conséquences directes d'une urbanisation croissante et donc la disparition rapide de terres arables au profit de constructions. Ce phénomène a pris une ampleur considérable sur les trente dernières années en particulier le long du front de mer et selon un axe est-ouest allant jusqu'à créer une jonction entre les centres villes historiques d'Alger et de Boumerdès distantes d'environ 50-60 km. La sélection de sept variables qui peut expliquer, en l'occurrence la distance aux routes, la distance à la mer, l'altitude (MNT), l'aspect, la pente, la distance aux terres agricoles, et 
finalement la distance au centre ville a conduit à l'élaboration de deux cartes prédictives respectivement de 2005 et 2013 et leur comparaison aux cartes de références correspondant aux mêmes dates montrent la concordance quasi parfaite des résultats (prédits et de référence). La précision atteint les 93\% et l'indice de Kappa gravite autour de 0.93 . Cette précision a tendance à baisser légèrement pour atteindre une valeur de $90 \%$ et un coefficient de Kappa de 0.89, marquant ainsi l'augmentation de l'erreur commise quant l'intervalle de temps entre les cartes prédites et de référence augmente. Cependant les résultats des tableaux (Fig. 4) confirment la validité des résultats (Kappa $>0.85$ ) et le degré de précision acceptable. La traduction cartographique des résultats de 2013 montre la poursuite de l'extension du bâti selon un axe SO, correspondant à l'embouchure de la plaine de la Mitidja et son ouverture sur la mer méditerranée.

Sur la figure 4, la photo de gauche représente les résultats pour 2013, celle de droite les résultats pour 2015.

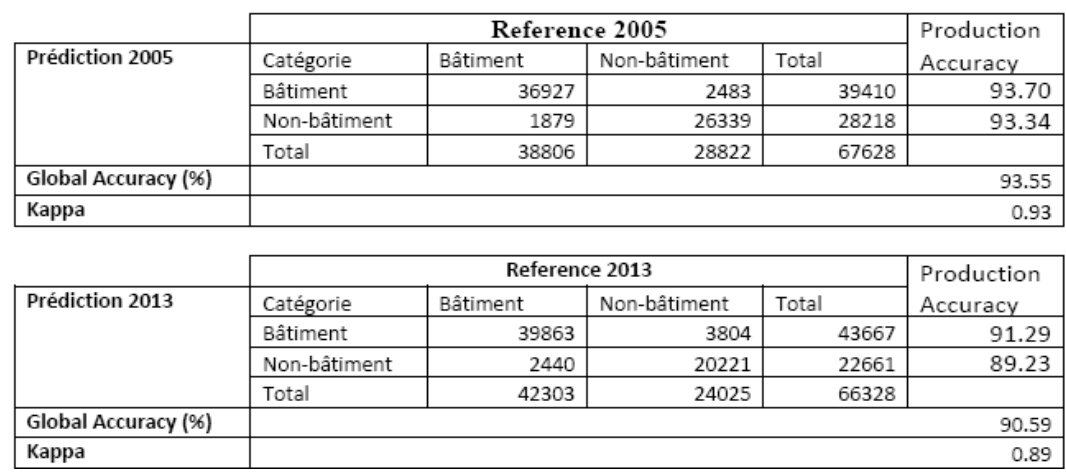



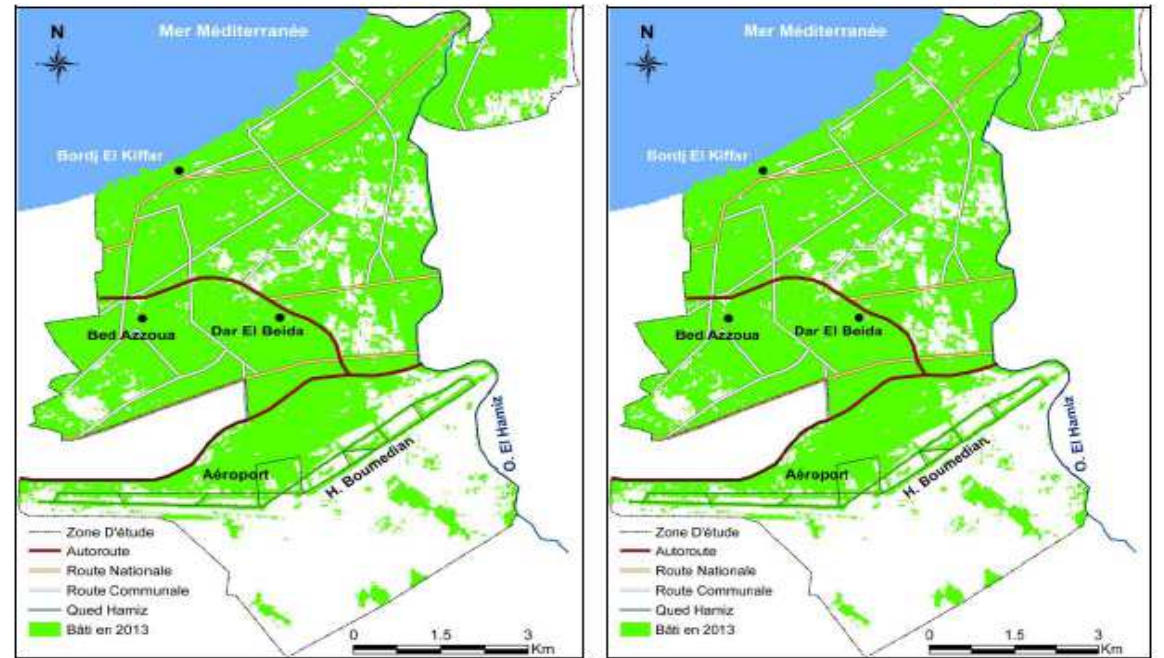

Fig. 4. matrice de confusion entre cartes prédictives de 2005 et 2013 avec les cartes de référence de 2005 et 2013

La présence de l'aéroport constitue une limite jusque là rigide qui commence à perdre de sa vigueur et devenir perméable. La cartographie prédictive de l'occupation de sols pour 2030 confirme la tendance générale à la hausse des surfaces bâties et la réduction massive des terres agricoles (Fig. 4). Elle montre également le saut entamé par le bâti vers le sud au-delà de l'aéroport, secteur qui se distingue par la forte présence de vergers et d'une agriculture maraichère. La construction d'un réseau routier à caractère autoroutier et donc la réduction des temps d'accès au centre ville d'Alger et aux nouveaux pôles urbains émergents (Ouled Fayet, Baraki, Khemis El Kechna) (seconde couronne algéroise) a favorisé considérablement la substitution des terres agricoles par des surfaces bâties.

La figure 5 montre les occupations de sol en 2005 et 2013 et donne les prévisions estimées pour les années 2020 (en violet) sur la carte de gauche et 2030 (en jaune) sur la carte de droite de la figure. La comparaison des deux cartes montre que les espaces interstitiels sont occupés progressivement par les constructions. Les rives de l'oued El Harrach ne sont pas épargnées ; les proximités de l'aéroport international d'Alger, autrefois protégé, ne le sont pas non plus. De manière générale la densité d'urbanisation augmente. 

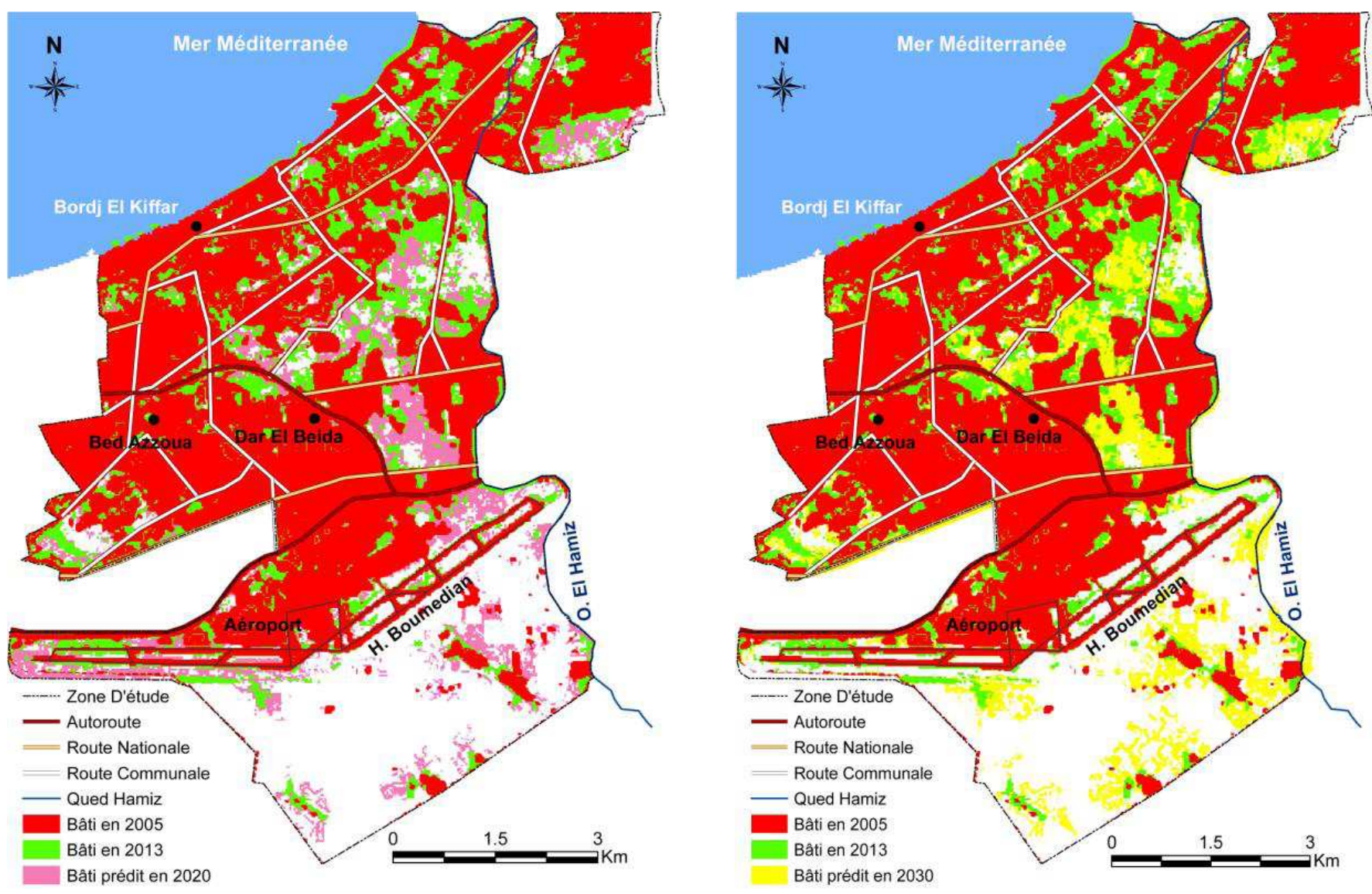

Fig. 5. Cartographie prédictive de l'occupation des sols pour 2030 et comparaison avec les années 2005 et 2013 
Sur la figure 5, la photo de gauche représente les résultats pour 2005 et celle de droite les résultats pour 2013. Sur la photo de droite, on notera (en jaune) l'occupation des interstices au nord et l'évolution du bâti à proximité de l'aéroport.

L'estimation de la consommation de l'occupation des sols sur la période 2005-2030 montre une tendance linéaire à la hausse jusqu'en 2020 des surfaces bâties. Ce processus observe un léger ralentissement jusqu'en 2030 marquant probablement l'entrée du département dans une phase de crise de foncier. La présence des monts de l'Ouarsenis (Chaine de Meftah-Larbaâ) constitue une barrière sud pouvant conduire à la modification de l'axe d'extension de la ville pour emprunter le grand axe de la Mitidja, en l'occurrence Est-Ouest, dédoublant ainsi l'axe historique frontalier qui caractérisait l'ancienne ville d'Alger. Ce schéma est amplement marqué par l'emprunte des grands axes autoroutiers récents.

Ainsi, la Mitidja dans sa partie Est perd près de 1000 ha dans cette zone de l'Algérois qui est couverte par une dizaine de communes; la figure 6 donne une estimation de l'évolution des surfaces agricoles et du bâti jusqu'en 2030.

\section{III.3. Discussions des résultats}

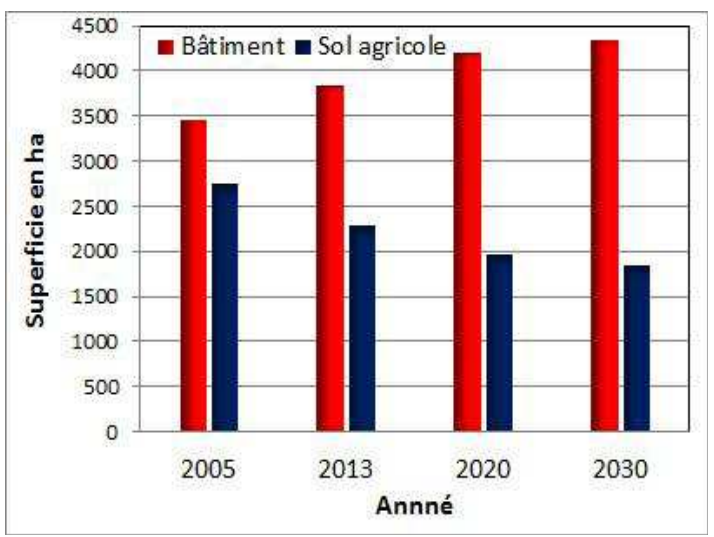

Fig. 6. Prévision comparée de l'occupation du sol par le bâti et l'agriculture de 2005 à 2030 
L'utilisation d'outils et de matériaux divers ont permis: i) de donner un aperçu de l'évolution démographique en Algérie de manière globale et de la wilaya d'Alger, où se trouve notre zone d'étude, de façon plus particulière ; ces résultats sont montrés sur le tableau 1, ii) de retracer l'historique de l'occupation du sol; la figure 2 résume cette progression entre 2005 et l'horizon 2030 ; on y notera l'augmentation des surfaces bâties (en rouge) et la diminution des surfaces agricoles, iii) de réaliser des cartes prédictives de l'occupation du sol et, surtout, de l'avancée permanent du bâti aux dépends des surfaces agricoles. Cette projection est réalisée pour 2020 dans un premier temps puis pour 2030. On notera, pour l'horizon 2030, que les environs de l'aéroport international commencent à se minéraliser. Parmi les résultats intéressants à relever, il nous faudra noter que l'étalement urbain à empiété sur 1283ha de terres cultivables en l'espace de 18 ans (1987-2005), soit 71ha par an; on constate ainsi une perte de terres agricoles de plus de $50 \%$ ( $52 \%$ selon nos calculs).

\section{Conclusion}

Malgré les limites mentionnées des deux méthodes de traitements liées à la subjectivité de l'interprétation et à la résolution des images satellitaires, elles ont permis d'obtenir des données quantitatives sur l'évolution spatio-temporelle de l'occupation du sol et l'évaluation de la consommation de l'espace agricole. Les résultats ont montré que la zone d'étude a perdu plus de 1200 ha de terre à haute potentialité agricole en l'espace de 18 ans à un rythme de 70ha par an, l'urbanisation mal maîtrisée a empiété sur la moitié de l'espace agricole durant la période 1987-2005. Deux causes principales peuvent être relevées :

i) Pour répondre aux besoins de la population en matière de logements, plusieurs programmes d'habitats collectifs et de lotissements individuels ont été approuvés et réalisés en détournant l'application des textes de protection du foncier agricole.

ii) La phase d'instabilité sécuritaire vécue par l'Algérie de 1990 à 2000 a engendré de nouvelles vagues d'exode rural et a amplifié ainsi la pression sur le foncier agricole. 
Les principaux résultats de la présente étude sont de deux ordres : i) une cartographie historique montre que l'espace agricole de la périphérie d'Alger est menacé par une urbanisation rapide et incontrôlée ; ii) une simulation prédictive de l'occupation de l'espace jusqu'en 2030, confirme cette tendance de la disparition graduelle des espaces agricoles ; iii) l'étude montre également une réorientation des axes historiques d'extension du SE vers le une direction EO l'envoyant sur la Mitidja.

Les causes d'une telle tendance nous paraissent être : i) l'influence du front de mer s'attenue après une période actuelle marquée par la préférence des espaces maritimes, ii) la mise en place de nouvelles ceintures autoroutières quadrillant l'algérois par le sud donne un nouveau schéma de la ville d'Alger sur le plan de son extension, enfin iii) l'émergence de plusieurs pôles urbains vers le sud au-delà de l'aéroport d'Alger marque un passage d'entrée du territoire dans une phase qui affiche les stigmates d'une pression foncière inévitable d'ici 2030.

\section{BIBLIOGRAPHIE}

Abdellaoui, Abdelkader (2007), Intégration de l'imagerie satellitale multi-résolution et de données terrain pour la réhabilitation des quartiers anciens en milieu oasien. Cas de la ville de Laghouat (Algérie) ; Analele Universității București Geografie ; 2007 ; pp. 5-20.

Abdellaoui, A. et Rougab, A. (1995), Caractérisation de la réponse du bâti : application au complexe urbain de Blida (Algérie). In Télédétection des milieux urbains et périurbains, Actes des VIe Journées scientifiques du Réseau Télédétection de l'AUPELF / UREF, 2- 5 octobre 1995, Liège (Belgique), pp. 75-83.

Bannari, A. (1996) : La télédétection et les indices de végétation pour la détection de la végétation éparse et moyennement dense : cas de l'environnement urbain; thèse de doctorat, Département de géographie et télédétection, Université de Sherbrooke, 230p.

Benblidia, N., Abdellaoui, A., Guessoum, A. et Bensaid, A, (2006), Utilisation de la morphologie mathématique pour l'analyse de l'occupation de l'espace en zones urbaines et périurbaines présaharienne. Cas de Laghouat (Algérie)", Revue Télédétection, vol. 6, no 2, pp. 177-190.

Ahmad, M.N. (1956) : Land utilisation work in Dacca district, East Bengal, Pakistan, 18 congrès international de géographie, Rio de Janeiro, Brésil, 1956.

Claramunt, C., Parent, C., Spaccapietra, S., Theriault, M. (1999), Database Modeling for environnemental and land use changes ; Geertmans, S., Openshaw, S., Stillwell, J. 
(eds), Geographical information and planning ; European Prospectives, Berlin ; Spinger.verlag, 1999, pp. 173-194.

Clibbon, P.B. (1965), L'évolution récente de l'utilisation du sol sur la rive nord du Saint-Laurent entre Québec et Montréal ; cahier de géographie du Québec 10(19), 1965 , pp. 55-71.

Coombs, D.B., Thie, J. (1979), The Canada Land Inventory System ; planning the uses and management of land, ASA, ESSA, Soil Society of America1979, pp. 909-933.

Dragicevic, S., Marceau, D.J. (2000), A fuzzy logic approach for modeling time in GIS international journal of geographic information science, 14, (3), 2000, pp. 225-245.

Dziewonski, M. (1956), detailed land use mapping in Poland; $18^{\text {ième }}$ congrès international de géographie ; Rio de Janeiro, Brésil, 1956.

European Environment Agency (2000), www.eea.europa.eu

George, Pierre (1957), Géographie Agraire ; Annales de Géographie ; 66, (363) ; 1957, pp. 27-31.

Gadal, S. (2003), Méthodes RSI pour l'identification des formes du bâti ; International SFTP conférence proceeding, Télédétection et photogrammétrie pour le développement en milieu urbain ; ENSG, Marne-la-Vallée, 26-28 novembre 2003.

Gribandi, D. et Albani, D. (1956), carte agraire de Frignano et de Lucarnie et de la Calibre ; $18^{\text {ième }}$ congrès international de géographie ; Rio de Janeiro, Brésil ; 1956.

Hornsby, K., Yuan, M. (2008), understanding dynamics of geographic domains, Boca Raton, FL:CRC Press, 2008.

Hung, P.T. (1998), Suivi de l'utilisation du sol à l'aide de l'image TM de Landsat et de la géomatique : le cas de district de Bao loc, provence de Loun dong, Vietnam (1992-1998), mémoire de maîtrise, département de géographie et de géomatique Sherbrooke ; université de Sherbrooke, 2000.

Jensen, J.-R. (1996), Introductory Digital Image Processing: A Remote Sensing Perspective. $2^{\text {eme }}$ edition, Prentice-Hall, Upper Saddle River, 318 p.

Langran, G. (1992), Time in geographical information systems, London, Taylor et Francis, 1992.

Theriault, M., Leraig, S., Gourmelon, F., Le Berre, I. (2011), Modélisation des changements d'occupation et d'utilisation du sol, cadres formels et exemple d'application ; revue internationale de géomatique, volume 21, n 3 / 2011.

Moller-Jensen, L. (1990), Knowledge-based classification of an urban area using texture and context information in Landsat- TM imagery. Photogrammetric Engineering and Remote Sensing, vol. 56, no 6, pp. 899-904.

Mun, L.C., Mc Clellan, J.B., Philpotts, L.E. (1966), Airphoto interpretation and rural land use mapping in Canada ; Photogrammetria, 21(3) ; pp. 65 -76, 1966.

Mutin, Georges (1997), La Mitidja, décolonisation et espace géographique ; édition CNRS, Paris, 1997.

Pagelow, M. (2004), Géomatique et géographie de l'environnement : De l'analyse spatiale à la modélisation prospective ; thèse $\mathrm{d}^{\prime}$ habilitation à diriger des recherches ; université de Toulouse le Mirail ; 2004.

Peuquet, D. (1994), A conceptual framework for the representation of temporal dynamics in geographic information systems; Annals of the association of American Geographers, 84 (3), pp. 441-461, 1994. 
Robin, M. (2002), Télédétection, Des satellites au SIG. Une analyse complète du processus de création d'un type essentiel d'information géographique. Nathan Université. 318 p., 2002.

Serendero, M.A. (1989), Extraction d'informations symboliques en imagerie SPOT : Réseaux de Communication et Agglomérations.

Thèse de Doctorat, Université de Nice, 1989.

Swain, P.H. et S.M. Davis (1978), Remote sensing: the quantitative approach. New York, McGraw-Hill, 396 p.

Serra, J. and Chermant, J.L. (1997), Mathematical morphology : A historical overview ; Acta stereological, vol. 16, n³, pp. 1-9.

Soille, P. (1999), Morphological Image Analysis. Springer-Verlag, Berlin-Heidelberg, 316 p.

Tomlinson, R.F. (1967), An introduction to the geo-information system of the Canada Land inventory , Otawa: ARDA , ministère des forets et du développement rural au Canada, 1967.

Wachowicz, M. (1999), Object oriented design for temporal GIS, London, Taylorand Francis, 1999. 